\title{
Esophagus Segmentation by Spatially-constrained Shape Interpolation
}

\author{
Andreas Fieselmann ${ }^{1,2}$, Stefan Lautenschläger ${ }^{2}$, Frank Deinzer ${ }^{2}$, \\ Matthias John' ${ }^{2}$ Björn Poppe ${ }^{1}$ \\ ${ }^{1}$ Medical Radiation Physics, University of Oldenburg, Oldenburg, Germany \\ ${ }^{2}$ Siemens AG Medical Solutions, Forchheim, Germany \\ andreas.fieselmann.ext@siemens.com
}

\begin{abstract}
The segmentation and visualization of the esophagus is helpful during planing and performing atrial ablation therapy to avoid esophageal injury. Only very few studies have addressed this segmentation problem which is challenging because the esophagus has a low contrast in medical images. In this work we present a technique to segment the esophagus based on the interpolation of Fourier descriptors of manually drawn contours. The interpolation is spatially-constrained using a dedicated correction term to avoid intersections with the convex shaped left atrial posterior wall. Our technique is fast, modality independent and achieves optimal results if at least three input contours are used. We validated our technique successfully with patient data and discuss the use of our technique in the clinical workflow.
\end{abstract}

\section{Introduction}

Atrial fibrillation can be treated by catheter ablation of the pulmonary veins inside the left atrium. During this intervention there is a small risk, however, to induce thermal injury to the adjacent esophagus. This can lead to the formation of an atrio-esophageal fistula and air from the esophagus is able to enter into the left atrium [1].

A strategy to avoid this often fatal complication is to decrease the power of the catheter at the contact area of the left atrium and the esophagus. Studies show that the esophagus is always in contact with the left atrium but its route varies widely [2]. Therefore, a segmentation of the esophagus before and during the intervention can help to plan the ablation strategy. The esophagus has a low contrast in cardiac CT, cardiac C-arm CT [3] and cardiac MRI images. This makes segmentation using edge-based techniques difficult.

Research on esophagus segmentation is very rare. Rousson et al. [4] propose a probabilistic shortest path approach to extract the esophagus from CT images. Their method requires a prior segmentation of the left atrium and the aorta. Two points on the centerline of the esophagus are needed as input to the algorithm which is computationally intensive. Ragan et al. [5] use deformable models to segment several organs but fail to accurately contour the esophagus. The work of Huang et al. [6] investigates optical flow to outline the esophagus and other 
organs. They construct initial 2D contours by Fourier interpolation which is a different concept than the interpolation of Fourier descriptors presented in section 2.1. Jeong and Radke [7] use Fourier descriptors to interpolate contours of the prostate in CT images and present a good overview about alternative shape interpolation techniques like triangulated meshes [8] and implicit functions [9].

In this work we present a technique to segment the esophagus that is independent on the type of modality. Our technique incorporates prior knowledge to avoid intersections with the left atrium. It is robust and faster than existing esophagus segmentation methods because it does not work directly on the image data.

\section{Materials and Methods}

There are two main assumptions in our segmentation technique. First, the shape of the esophagus contour changes smoothly between axial slices. Second, the esophagus does not intersect with other structures like the left atrium.

We model the esophagus by a sequence of contours along the longitudinal axis (patient axis). As input to our algorithm we expect at least two esophagus contours and a prior segmentation of the left atrium. In the clinical workflow the left atrium is the primary organ of interest and always segmented before the esophagus, so this requirement is not a limitation of our algorithm. The contours between the input contours are determined by a slice-by-slice interpolation in the frequency domain and a correction method is used to ensure that the contours do not intersect with the left atrium. The concept of Fourier descriptors and our interpolation technique are presented below.

\section{$2.1 \quad$ Fourier Descriptors}

Fourier descriptors are used to describe the shape of $2 \mathrm{D}$ closed curves [10]. They are constructed as follows. A discrete closed curve with coordinates $\left(x_{n}, y_{n}\right)$ and $n=0,1, . ., N-1$ can be converted into a complex-valued periodic function $a_{n}$ by the mapping $\mathbb{R}^{2} \mapsto \mathbb{C}: a_{n}=x_{n}+j \cdot y_{n}$ with the imaginary unit $j$. The discrete Fourier transform $\mathcal{F}$ in $(1)$ is used to transform $a_{n}$ into the frequency domain. Its inverse transform $\mathcal{F}^{-1}$ is given in (2).

$$
\begin{aligned}
& A_{k}=\mathcal{F}\left\{a_{n}\right\}=\frac{1}{N} \sum_{n=0}^{N-1} a_{n} \cdot \exp \left(-\frac{j 2 \pi k n}{N}\right) \\
& a_{n}=\mathcal{F}^{-1}\left\{A_{k}\right\}=\sum_{k=0}^{N-1} A_{k} \cdot \exp \left(\frac{j 2 \pi k n}{N}\right)
\end{aligned}
$$

The coefficients $A_{k}$ with $k=0,1, . ., N-1$ are called the Fourier descriptors of the curve. The zeroth descriptor $A_{0}$ contains the center of mass of the curve and all other descriptors are invariant to translation. Fourier descriptors are wellsuited to describe elliptical shaped curves whose smoothness can be controlled by 
filtering of the high-frequency coefficients. We keep 6 Fourier descriptors in the following analyses. In order to compute the Fourier descriptors we first resample the curve in the spatial domain by piecewise linear interpolation such that the number of points is the same for all curves.

\subsection{Spatially-Constrained Shape Interpolation}

The Fourier descriptors $A_{k, z}$ describe the esophagus contour in the axial plane with the longitudinal coordinate $z$. The shapes of two input contours $A_{k, z_{1}}$ and $A_{k, z_{2}}$ with $z_{2}>z_{1}$ are interpolated piecewise-linearly in steps of $d z$ starting from $A_{k, z}=A_{k, z_{1}}$ according to (3).

$$
A_{k, z+d z}=A_{k, z}+d z \cdot \frac{A_{k, z_{2}}-A_{k, z}}{z_{2}-z}
$$

After each iteration step the new contour is transformed back into the spatial domain using (2) and checked if it intersects with the left atrium. If the contour intersects it is corrected as follows.

Let $\boldsymbol{r}_{i}(i=1, . ., M)$ denote the vectors from the center of mass of the contour to the intersecting points. The correction vector $\boldsymbol{t}$ defined in (4) points in the opposite direction of the vectorial sum of all $\boldsymbol{r}_{i}$ (Fig. 1) and is normalized to one pixel length.

$$
\boldsymbol{t}=\left(\begin{array}{c}
t_{x} \\
t_{y}
\end{array}\right)=-\frac{1}{M} \sum_{i=1}^{M} \boldsymbol{r}_{i}
$$

In the spatial domain all contour points shifted by $\boldsymbol{t}$. In the frequency domain only $A_{0, z}$ is changed by adding $t_{x}+j \cdot t_{y}$. If the contour still intersects then the vectors $\boldsymbol{r}_{i}$ are updated and the procedure is repeated. All input contours are sorted with respect to their longitudinal coordinate and the interpolation is performed between each neighboring pair.

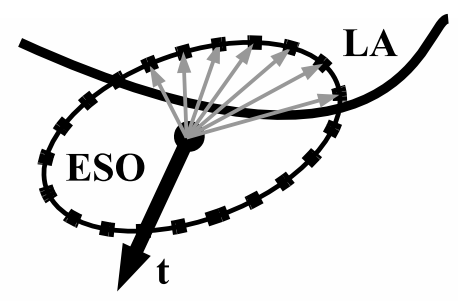

Fig. 1. Intersection of the esophagus (ESO) and the left atrium (LA) generates a correction vector $t$

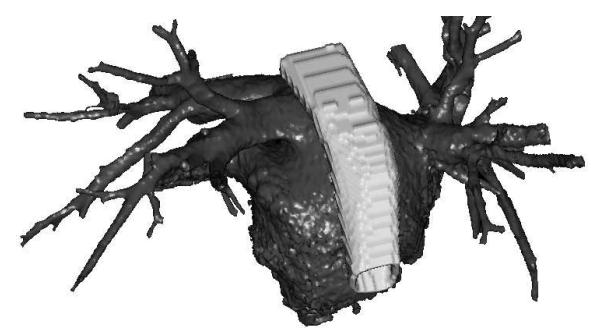

Fig. 2. 3D Volume rendering of the segmented esophagus (light) and the left atrium (dark) 
Table 1. Comparison of segmentation results using 2, 3 or 5 input contours. A dice coefficient value $C_{D}=1$ indicates complete agreement with the ground truth

\begin{tabular}{lllllllll}
\hline patient number & 1 & 2 & 3 & 4 & 5 & 6 & 7 & 8 \\
\hline$C_{D}(2$ contours $)$ & 0.60 & 0.61 & 0.67 & 0.76 & 0.49 & 0.40 & 0.61 & 0.65 \\
$C_{D}(3$ contours $)$ & 0.84 & 0.70 & 0.76 & 0.80 & 0.79 & 0.51 & 0.80 & 0.81 \\
$C_{D}(5$ contours $)$ & 0.89 & 0.81 & 0.84 & 0.85 & 0.86 & 0.74 & 0.86 & 0.88 \\
\hline
\end{tabular}

\section{Results}

In order to validate our technique we segment the esophagus in $8 \mathrm{CT}$ scans of patients treated with atrial catheter ablation. In each volume the left atrium is segmented using the software syngo ${ }^{\circledR}$ InSpace EP (Siemens AG Medical Solutions) and an expert manually draws 9 esophagus contours in equally spaced axial slices. We assume that a segmentation using all 9 contours is a precise segmentation ("ground truth"). We choose 9 contours because it is possible to extract subsets of 2, 3 and 5 equally spaced contours. Then we perform the segmentation using the contours from each subset as input to the algorithm. The dice similarity coefficient $C_{D}$ [11] defined in (5) is a measure to compare the segmentation results. $|A|$ is the volume of the segmentation with 9 input contours, $|B|$ is the volume of the segmentation with 2,3 or 5 input contours respectively and $|A \cap B|$ is the intersection of $|A|$ and $|B|$.

$$
C_{D}=\frac{2 \cdot|A \cap B|}{|A|+|B|}
$$

The segmentation of the esophagus using all 9 input contours has been assessed by visual inspection and it is very consistent with the true position of the esophagus in all patient data. The results from Table 1 and Fig. 3 show that 3 input contours produce an optimal segmentation with a median dice coefficient of about 0.8 which is similar to the value in [4]. A dice coefficient value greater than 0.7 is usually considered as a good agreement [4]. Two contours may be sufficient for an approximate localization of the esophagus. A volume rendering of the esophagus segmented from 3 input contours is shown in Fig. 2. The time to draw 3 input contours and perform the computations is about 15 seconds.

\section{Discussion}

Our technique can be used to plan the ablation strategy with cardiac CT scans and is also applicable with cardiac C-arm CT data during the intervention. In order to be modality independent our algorithm does not work directly on the image data. The technique is robust because the input contours profit from human expert knowledge and our correction term avoids intersections with the left atrium. The time needed to segment the esophagus including user interactions is about 15 seconds which is adequate for the clinical workflow and less than other, fully automated techniques usually require. The computational time for the pure 
Fig. 3. Shape interpolation of the esophagus contour (thick lines). Two input contours (thin lines) are drawn in (a) and (d). The shown slices are equally space, start at the caudal side of the left atrium (a) and end at the atrial center (d)

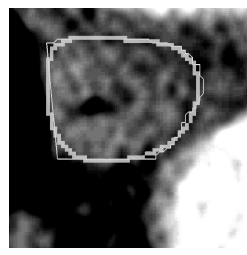

(a)

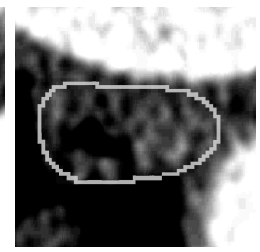

(b)

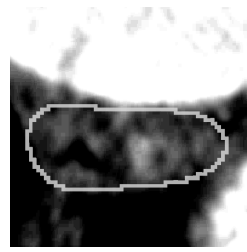

(c)

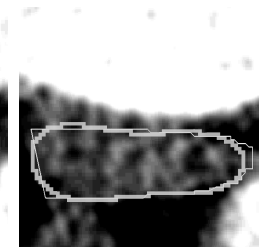

(d)

algorithm is less than a second. The chosen interpolation scheme is well suited for elliptical structures like the esophagus and therefore gives visually pleasing and precise results with only a few input contours. We are currently working on extensions of our technique to find input contours automatically by an algorithm. E.g. air holes inside the esophagus are visible in CT images and can be used to localize the esophagus and detect the esophagus contour.

\section{References}

1. Pappone C, Oral H, Santinelli V, et al. Atrio-esophageal fistula as a complication of percutaneous transcatheter ablation of atrial fibrillation. Circulation. 2004;109(22):2724-26.

2. Cury RC, Abbara S, Schmidt S, et al. Relationship of the esophagus and aorta to the left atrium and pulmonary veins: Implications for catheter ablation of atrial fibrillation. Heart Rhythm. 2005;2(12):1317-23.

3. Lauritsch G, Boese J, Wigström L, et al. Towards cardiac C-arm computed tomography. IEEE Trans Med Imaging. 2006;25(7):922-34.

4. Rousson M, Bai Y, Xu C, et al. Probabilistic Minimal Path for Automated Esophagus Segmentation. Proc SPIE. 2006;6144:49-4H.

5. Ragan D, Starkschall G, McNutt T, et al. Semiautomated four-dimensional computed tomography segmentation using deformable models. Med Phys. $2005 ; 32(7): 2254-61$.

6. Huang TC, Zhang G, Guerrero T, et al. Semi-automated CT segmentation using optic flow and Fourier interpolation techniques. Comput Methods Programs Biomed. 2006;84(2-3):124-34.

7. Jeong Y, Radke RJ. Reslicing axially sampled 3D shapes using elliptic Fourier descriptors. Med Image Anal. 2007;11(2):197-206.

8. Boissonnat JD. Shape reconstruction from planar cross sections. Computer Vis Graph Image Process. 1988;44(1):1-29.

9. Turk G, O'Brien JF. Shape Transformation Using Variational Implicit Functions. Comput Graph (ACM). 1999;33:335-42.

10. Zahn CT, Roskies RZ. Fourier descriptors for plane closed curves. IEEE Trans Comp. 1972;C-21(3):269-81.

11. Heimann T, Thorn M, Kunert T, et al. Empirische Vergleichsmaße für die Evaluation von Segmentierungsergebnissen. Proc BVM. 2004; p. 165-9. 